\title{
Time- and Spectrally-Resolved PL Study of a Regular Array of InP/InAs/InP Core-multishell Nanowires
}

\author{
B. Pal, ${ }^{1, \text { * }}$ K. Goto,${ }^{1}$ M. Ikezawa,,${ }^{1}$ Y. Masumoto, ${ }^{1}$ P. Mohan, ${ }^{2}$ J. Motohisa,${ }^{2}$ and T. Fukui ${ }^{2}$ \\ ${ }^{1}$ Institute of Physics, University of Tsukuba, Tsukuba 305-8571, Japan \\ ${ }^{2}$ Research Center for Integrated Quantum Electronics, Hokkaido University, Sapporo 060-8628, Japan
}

(Dated: October 8, 2018)

\begin{abstract}
Time- and spectrally-resolved PL from a periodic array of InP/InAs/InP core-multishell nanowires is presented. InAs layer shows multipeak PL spectra. PL decay is nonexponential and very slow, with decay rate depending on energy.
\end{abstract}

Nanometer-scale semiconductor heterostructures such as quantum dots (0D), quantum wires (1D), and quantum wells $(2 \mathrm{D})$ have been interesting research target due to their unique size dependent electronic and optical properties associated with the lower dimensionality and quantum confined effect. There have been revived interests in the semiconductor nanowires due to the recent success in the growth and fabrication of regular array of core-shell and core-multishell nanowires (CMNs) $, 1,2$ Such nanowires can be used as building blocks of sophisticated nanoscale electronic and photonic devices because of their capability to function as both device element as well as the interconnecting wires for the devices. Incorporation of heterostructures into the nanowires opens up the possibility to build multifunctionality into a single device. Though much efforts have been devoted to the growth and fabrication of periodic array of uniformly grown CMNs, carrier dynamics in such structures has not been studied so far by time-resolved optical spectroscopy.

In this paper we report our study of time- and spectrally-resolved photoluminescence (PL) from a regular array of $\mathrm{InP} / \mathrm{InAs} / \mathrm{InP} \mathrm{CMNs}$, in which the InAs layer acts as a strained quantum well $(\mathrm{QW})$ embedded in the higher bandgap InP material. PL spectra from the InAs layer show multiple peaks due to monolayer (ML) scale variation in the layer thickness over the CMN array. Decay rate measured within a PL peak is found to be energy dependent, suggesting the presence of spectral diffusion due to inhomogeneous broadening. The PL decays nonexponentially showing very slowly decaying component surviving beyond $100 \mathrm{~ns}$. This behavior may be resulted from the spreading of the electron wavefunction of the very thin InAs layer into the InP barriers.

The sample studied here is a periodic array of uniformly grown, vertically oriented $\mathrm{InP} / \mathrm{InAs} / \mathrm{InP} \mathrm{CMNs}$ grown by using selective area metalorganic vapor phase epitaxy. Each nanowire has a hexagonal-cylindrical symmetry and it consists of an inner InP core and InAs and InP inner and outer shells, respectively, in which the InP core and the $\mathrm{InP}$ outer shell serve as the barrier layers while the InAs shell acts as a strained QW layer [see Fig. [1-inset]. Further details of the sample structure and growth procedure may be found in Ref. 2 .

The PL spectra from this sample are measured at $2 \mathrm{~K}$ for excitation above and below the InP barrier by a Ti:Sapphire laser. A monochromator with a spectral resolution of about $1 \mathrm{meV}$, a liquid $N_{2}$-cooled InGaAsP photomultiplier tube, and a photon counter is used. The focused laser spot size on the sample was about $150 \mu \mathrm{m}$ in diameter covering about 3000 nanowires. The highest energy peak observed in Fig. 1 for above barrier excitation is associated with the InP barrier. The remaining peaks observed for both above and below barrier excitation can be assigned to the InAs QWs. The spectra are measured from an ensemble of CMNs. In the ensemble, possibly there are monolayer scale variations in the thickness of InAs QW layer. From a calculation of the ground-state transition energy in strained InAs QWs with InP barriers, the main three peaks are assigned to $1 \mathrm{ML}, 2 \mathrm{ML}$, and $3 \mathrm{ML}$ wide QWs, respectively, from higher to lower energy: 2

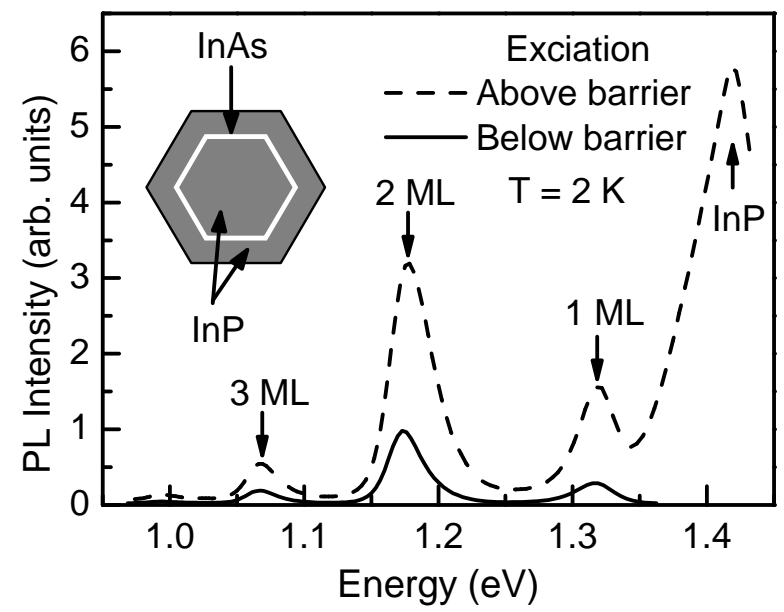

FIG. 1: Schematic cross-section of a nanowire and PL spectra of the InAs layer for excitation above and below the InP barrier.

The PL decay for the InAs peaks is measured over a 2 ns time-span by using a synchroscan infrared streak camera and a picosecond Ti:Sapphire laser with $82 \mathrm{MHz}$ repetition rate. The data is plotted in Fig. 2, showing very similar decay rates for all three peaks. In the 2 ns time-span the decay looks like an exponential, giving about 4 ns decay time. However, presence of a large PL signal at negative times before the arrival of the excitation pulse suggests that very long decay components are present. 


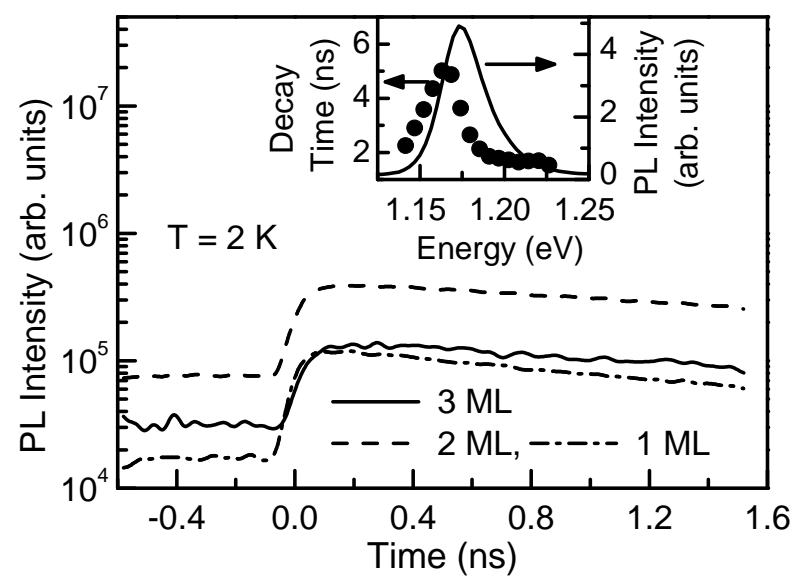

FIG. 2: PL decay at all three peaks of InAs QWs. Inset: Energy dependence of the PL decay time for the $2 \mathrm{ML}$ peak.

We find that for a given peak, the decay rate depends on the spectral energy. We systematically measure the PL decay at different spectral energy within the $2 \mathrm{ML}$ peak. A dependence of PL decay time on the spectral position is shown in the inset of Fig. 2. A two-dimensional plot of the grayscale coded PL intensity on to the timeenergy surface for the $2 \mathrm{ML}$ peak in Fig. 3 shows that the spectral weight is shifted towards the lower energy at increased time. This suggests the presence of spectral diffusion due the inhomogeneous broadening present in the sample. This may be resulted from the inhomogeneous strain in the QWs in the ensemble of CMNs. ${ }^{2}$

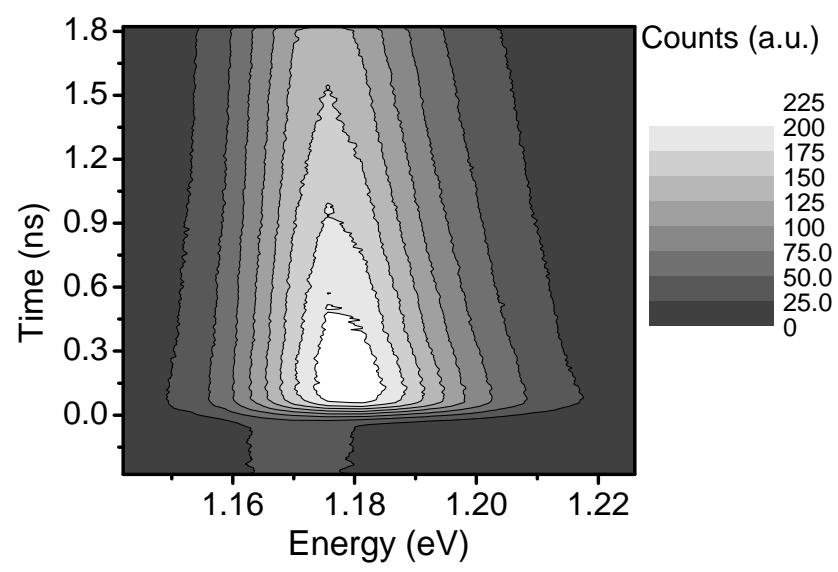

FIG. 3: Two-dimensional plot of PL intensity (grayscale coded) on the time-energy surfaces.
To investigate the PL decay over a longer time-span we reduce the laser repetition rate to $800 \mathrm{kHz}$ by using a pulse picker. Then the PL decay is measured by using the time-correlated single photon counting technique. Figure 4 shows the PL decay over a 200 ns time-span for the 1,2, and 3 ML peaks of the InAs QWs. The measured time-profile of the laser pulse is also shown, giving a time resolution of about 1 ns. The PL decay is nonexponential and very long components surviving beyond $100 \mathrm{~ns}$ are present.

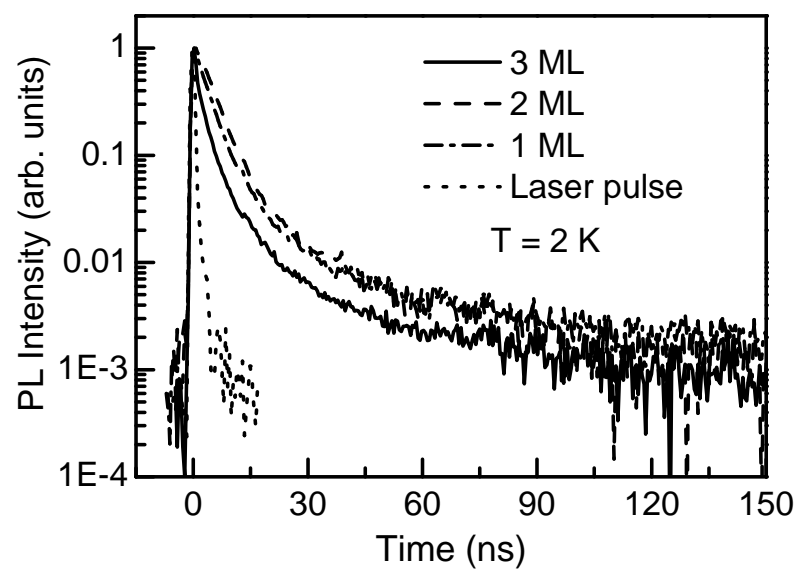

FIG. 4: Nonexponential PL decay extending over $100 \mathrm{~ns}$. Measured laser pulse profile is also shown.

To understand the origin of the slow decay of PL we may consider the following. For very thin InAs QWs, the electron state is not very well confined and there may be appreciable spreading and penetration of the QW electron wavefunction into the barrier layer $\stackrel{3}{-}$ In a way, the PL can be considered as the indirect transition in a typeII material. In such a case, slow and nonexponential decay of PL is reported in the literature. ${ }^{4}$ The slow decay is a result of insufficient overlap of the electron and hole wavefunctions. The nonexponential decay results from a distribution of decay rates. Further study by theoretical modelling and experiments to measure the temperature dependence of the decay rate and PL intensity is in progress to clarify the issue.

In summery, time- and spectrally-resolved PL from a periodic array of $\mathrm{InP} / \mathrm{InAs} / \mathrm{InP}$ core-multishell nanowires is presented. InAs layer shows multipeak PL spectra. PL decay is nonexponential and very slow, with decay rate depending on energy.
* E-mail: bipulpal@sakura.cc.tsukuba.ac.jp

1 See e.g., L. J. Lauhon, M. S. Gudiksen, D. Wang, and C. M. Lieber, Nature 420, 57 (2002).

2 P. Mohan, J. Motohisa, and T. Fukui, Appl. Phys. Lett. 88, 133105 (2006).
${ }^{3}$ U. Cebulla, G. Bacher, A. Forchel, G. Mayer, and W. T. Tsang, Phys. Rev. B 39, 6257 (1989).

${ }^{4}$ See e.g., F. Minami, K. Hirata, K. Era, T. Yao, and Y. Masumoto, Phys. Rev. B 36, 2875 (1987). 